\title{
Revisiting path steering for 3D manipulation tasks
}

\author{
Lei Liu ${ }^{\mathrm{a}, *}$, Jean-Bernard Martens ${ }^{\mathrm{b}}$, Robert van Liere ${ }^{\mathrm{a}}$ \\ ${ }^{a}$ CWI, Amsterdam, The Netherlands \\ ${ }^{\mathrm{b}}$ Eindhoven University of Technology, The Netherlands
}

Received 31 May 2010; received in revised form 22 November 2010; accepted 23 November 2010

Communicated by D.A. Bowman

Available online 27 November 2010

\begin{abstract}
The law of path steering, as proposed by Accot and Zhai, describes a quantitative relationship between the human temporal performance and the path spatial characteristics. The steering law is formulated as a continuous goal-crossing task, in which a large number of goals are crossed along the path. The steering law has been verified empirically for locomotion, in which a virtual driving task through straight and circular paths was performed.

We revisit the path steering law for manipulation tasks in desktop virtual environments. We have conducted controlled experiments in which users operated a pen input device to steer a virtual ball through paths of varying length, width, curvature and orientation. Our results indicate that, although the steering law provides a good description of the overall task time as a function of index of difficulty $I D=L / W$, where $L$ and $W$ are the path length and width, it does not account for other relevant factors. We specifically show that the influence of curvature can be modeled by a percentage increase in steering time, independent of index of difficulty. The path orientation relative to the viewing direction has a periodic effect on the steering time, which can be optimally described by a function of Fourier series expansions. In addition, there is also an effect of the handedness of the subjects on the steering between the left and right districts in 3D manipulation tasks. (c) 2010 Elsevier Ltd. All rights reserved.
\end{abstract}

Keywords: Virtual reality; Path steering; User study

\section{Introduction}

Path steering is a primitive interaction task that requires a user to navigate through a path of a given length and width. Navigating through nested-menus, drawing curves within boundaries, and locomotion along a predefined track are just a few examples of interaction tasks that can be thought of as steering tasks. Recently, Accot and Zhai have proposed the law of steering as a law that describes a quantitative relationship between the human temporal performance and the path spatial characteristics (Accot and Zhai, 1997; Zhai et al., 2004). The law was empirically verified to model human locomotion in virtual reality, in which case users

\footnotetext{
*Corresponding author. Tel.: + 31626998706; fax: + 31205924199 .

E-mail addresses: Lei.Liu@cwi.nl, derekliulie@gmail.com (L. Liu), j.b.o.s.martens@tue.edu (J.-B. Martens),

Robert.van.Liere@cwi.nl (R.van Liere).
}

were exposed to a virtual driving simulator to drive a virtual vehicle on straight and circular paths.

In the same paper (Zhai et al., 2004), Accot and Zhai posed the question if the steering law could also be used to model the performance in 3D manipulation tasks. The "ring and wire" task was given as an example, i.e. a user navigates a torus with a 6DOF (degrees of freedom) input device across a (curved) trajectory. Note that this task requires both the position and the orientation of the ring, which is substantially more complex than steering the position of a point in a plane. Since 3D manipulation tasks are very different from virtual driving tasks, it is well worth investigating how the steering law can be applied to such tasks. In order to limit the complexity, we propose to decouple the steering of position and orientation, which will result in an alternative interaction task from the "ring and wire" task. Gaining a more fundamental understanding of the steering movement in such a task is also likely to result in design rules that are useful for the development of higher level interactive desktop manipulation techniques. 
In a previous paper (Liu et al., 2010), we studied the path steering for 3D manipulation tasks in a virtual environment. Our goal was to verify the steering law and, if appropriate, identify other relevant factors that affect users' performance for such tasks. This was achieved by conducting two experiments of within-subject design, in which users operated a pen input device to navigate a virtual ball through paths of varying length, width, curvature and orientation. The first experiment focused on how path curvature affects the steering movement. For this, paths of different curvatures were used (see Fig. 1, top). The second experiment focused on the effects of path orientation with respect to the viewing direction (Fig. 1, bottom). Path orientation was determined by two angles: one angle specifying the rotation around the $y$-axis, and the other specifying the rotation around the $z$-axis. As indicated by the experimental results, the influence of path curvature could be successfully modeled by a percentage increase in steering time, independent of path length and width. The effect of path orientation relative to the viewing angle, however, was not quantitatively expressed in the model due to an insufficiency of the data. We only qualitatively demonstrated that the influence of path orientation had an asymmetric V-shaped performance on steering time and that the time of steering to the left is different from to the right.

In this work, we extend our previous study by conducting a new experiment to further examine the effect of path orientation with respect to the viewing direction on the steering time. Our aim is to, if possible, model the steering time as a function of path orientation, together with path length, width and curvature. The consequence is that the human temporal performance of doing a path steering task can be quantitatively expressed as a composite effect of all spatial characteristics that influence the task. As Fitts' law (Fitts, 1954) has been extensively used to compare the temporal performance of pointing tasks (MacKenzie et al., 1991; MacKenzie, 1992; Worden et al., 1997; Liu and van Liere, 2009), the new law can serve as a quantitative approach to evaluate the performance and

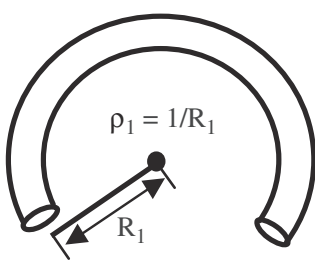

Path 1

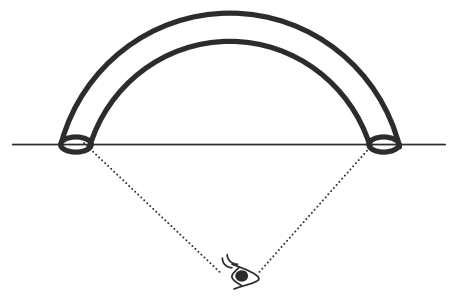

Fig. 1. Two paths of the same length and width, but of different curvatures (top). The same path in different orientations (bottom). efficiency between, e.g. input devices or interaction techniques for path steering tasks.

For completeness, some of the material (basically Experiment 1) in the previous study (Liu et al., 2010) is repeated. The contributions of the whole paper are summarized as follows:

- We experimentally demonstrate that the steering law provides a good description of the overall task time as a function of index of difficulty $I D=L / W$, where $L$ and $W$ are the path length and width.

- We demonstrate that the influence of path curvature can be modeled by a percentage increase in steering time, independent of index of difficulty.

- We demonstrate that the path orientation relative to the viewing direction has a periodic effect on path steering, which can be modeled with Fourier series expansions.

- We also demonstrate that subjects' handedness plays a part in the steering of paths with varying orientations.

Whereas the new contributions of this extended work focus on the last two items.

\section{Modeling pointing and steering}

The pointing tasks have been well studied (e.g. Boritz and Booth, 1997; Grossman and Balakrishnan , 2004). The bestknown law for modeling the user performance of pointing movements is Fitts' law (Fitts, 1954; MacKenzie, 1992), which predicts movement time of a pointing task as a function of the distance from source to target and the size of the target. One common formulation of Fitts' law is

$T=a+b \log _{2}\left(\frac{L}{W}+1\right)$

where $a$ and $b$ are experimentally determined constants, $L$ is the distance to the target, and $W$ is the target width. The expression $I D=\log (L / W+1)$ is referred to as the index of difficulty of the task. Although initially formulated from a 1D experiment, Fitts' law has been extended to model 2D (MacKenzie and Buxton, 1992) and 3D (Murata and Iwase, 2001) pointing tasks, respectively. In the previous papers (Liu et al., 2009; Nieuwenhuizen et al., 2009), we have shown that the $3 \mathrm{D}$ pointing movement in virtual reality can often be modeled by a two-component model (Meyer et al., 1988): an initial ballistic movement followed by a perceptually guided corrective movement. The conclusion was that, although Fitts' law is an excellent description of total movement time, the two-component model can give more insight into the users' performance during the movement. In a recent research, Fitts' law was adapted for distal pointing (raycasting) tasks (Kopper et al., 2010), in which the index of difficulty of the tasks was described as a function of the angular amplitude of movement and the angular size of the target.

The steering law, as developed by Accot and Zhai, was derived from the idea that a steering task can be broken 
down into a large number of segments, each of which can be treated as a goal-crossing task with the same index of difficulty. The total movement time can then be modeled by Fitts' law, with the $I D$ for the whole task calculated by the sum of all the $I D$ s of the segments. If the path width varies along the path, the generic steering law is expressed by the following equation:

$T_{C}=a+b I D=a+b \int_{C} \frac{d s}{W(s)}$

where $a$ and $b$ are empirically determined constants, $C$ is a curved path, $s$ is the elementary path length along $C$ and $W(s)$ is the path width at path length $s$. In those cases where path width is constant along the path, the steering law can be rewritten as

$T_{C}=a+b \frac{L}{W}$

with $L$ and $W$ representing the length and the width of the path, respectively.

It is important to note that the steering law implies that movement time is only a function of the length and the width of the path. This relationship is counterintuitive, since it would seem that the shape of a path should also influence the movement time. One example is that when driving from a straight road onto a roundabout, we usually have to slow down and the smaller the roundabout, the slower the more likely we are to reduce our speed. Fig. 1 (top) shows two paths of the same length and width, but of different constant curvatures. Intuitively, it is easier and faster to steer through path 2 than through path 1.

The steering law was initially formulated and developed to model 2D steering tasks for desktop computers with 2DOF input devices. It would seem that in $3 \mathrm{D}$ world, path orientation is also likely to affect the performance. For example, a path tracing movement in the $z$-direction might be very different from the same movement in the $x$-direction (see Fig. 1, bottom).

Finally, it should be noted that, although the law of pointing (Eq. (1)) and the law of steering (Eq. (3)) seem somewhat similar, they essentially describe very different movements. In Eq. (1), the index of difficulty is described by a logarithmic term, whereas in Eq. (3), the relationship is linear. We will get back to this point in the discussion section.

Many HCI researchers have applied the steering law to model interaction. Examples include examining scale effects by adjusting the input device's Control-Display ratio (Accot and Zhai, 2001), similar to semantic pointing (Blanch et al., 2004; Elmqvist and Fekete, 2008) for pointing tasks, evaluating the performance of multifarious input devices (Accot and Zhai, 1999) and investigating steering around sharp corners (Pastel, 2006). Besides, Naito et al. (2004) has extended the application of steering law to an environment of "spatially couple style", while Grossman et al. (2006) has confirmed its validity on the Hover-widget-based steering task using pen-operated devices. However, these studies were restricted to $2 \mathrm{D}$ desktop environments with $2 \mathrm{DOF}$ input devices. A notable exception is Kattinakere et al.
(2007), who conducted a series of experiments to validate the steering law in table top environments. Above-the-surface layers of certain thickness were used to constrain steering movements above the tabletop, a similar way of bringing "target height" into Fitts' law (Accot and Zhai, 2003). Our study differs from these studies in a number of ways. We investigate the steering law in a head tracked virtual environment using 6DOF input devices. Our environment is not co-located, i.e. there is an offset between the physical input device and the virtual representation of the pen. The curved paths we consider are less constrained than the constrained shape of paths in most other studies.

\section{Experiment}

For our experiments, we have designed the ball and tunnel task, as it is a more straightforward extension of the $2 \mathrm{D}$ steering law than the ring and wire task proposed by Accot and Zhai. Using an input stylus, the user pushes a virtual target ball through a tunnel. The target ball is constrained to the boundary of the tunnel, so that the width of the tunnel is defined by the diameter of the target ball. The steering path width is defined as the amplitude of the cursor ball with which the cursor ball is in contact with the target ball, i.e. the tunnel width plus two times cursor ball radius (see Fig. 2). The visual feedback of the stylus consists of a pen with a small cursor ball on the tip of the pen. The visual feedback of the target ball is used as the progress indicator for the task.

The goal of the task is to push the target ball from one end of the tunnel to the other end as fast as possible. To push the target ball, two requirements must be met:

1. The cursor ball must intersect the tunnel.

2. The cursor ball must be in contact with the target ball.

If one of these requirements fails, e.g. the cursor is not within the boundary of the path, then the user must correct for this by returning the cursor to the tunnel and continuing the task where she left off. A task starts when the target ball departs from one end of the tunnel and it continues until the target ball reaches the other end of the tunnel.

\subsection{Environment and apparatus}

The experimental setup was performed in a head tracked stereoscopic desktop virtual environment with a 67 -inch

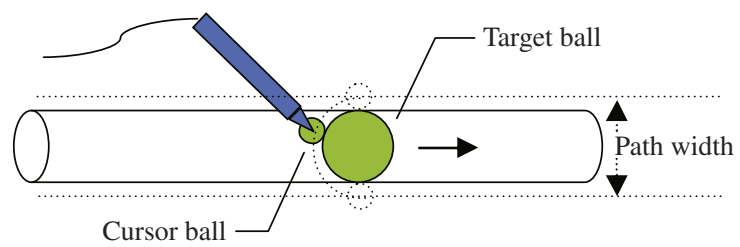

Fig. 2. Ball and tunnel task: a cursor ball pushes a target ball through a tunnel. Tunnel width $=$ diameter of target ball; steering path width $=$ tunnel width $+2 \times$ radius of cursor ball $=2 \times$ (radius of target ball + radius of cursor ball). 
display (Fig. 3). Subjects were seated $1.35 \mathrm{~m}$ from the display and were required not to rest their arms on the table (Fig. 4). The origin of the visual space was $0.4 \mathrm{~m}$ in front of the display and 0.6 above the desktop, while the origin of the motor space was set to $1.05 \mathrm{~m}$ in front of the display and $0.3 \mathrm{~m}$ above the desktop. The Control-Display ratio was always kept as 1 .

As shown in Fig. 3, the tunnel was drawn as a semitransparent 3D tube through which the cursor ball and the target ball could be easily seen. To enhance the depth perception, we used a stereoscopic viewing, a head tracking, a head lighting and a $0.72 \mathrm{~m} \times 0.4 \mathrm{~m} \times 0.4 \mathrm{~m}$ wire-frame box with a chessboard floor in the virtual world.

For the specific apparatus, we used a desktop PC with high end GPU, a Samsung HL67A750 3D-capable LED DLP HDTV, a pair of Crystal Eyes stereoscopic LCD

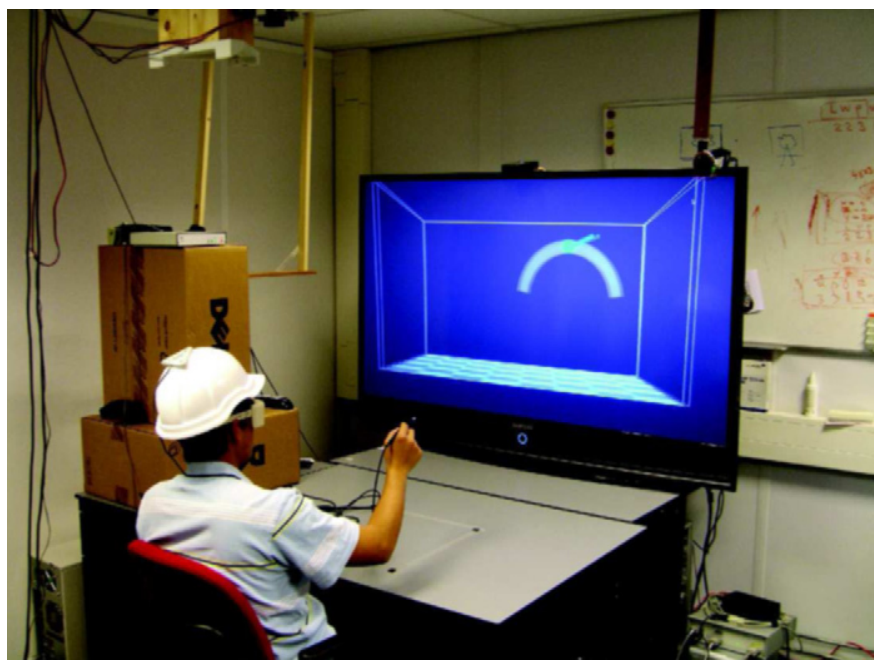

Fig. 3. The experimental environment: a head tracked stereo display and a $6 \mathrm{DOF}$ input stylus. glasses, a Polhemus FASTRAK connected by one 6DOF stylus tracker, and an ultrasound Logitech of 6DOF for head tracking. The resolution of the display was set to $1920 \times 1080 @ 120 \mathrm{~Hz}$. The end-to-end latency was measured to be approximately $80 \mathrm{~ms}$ using the method proposed by Steed (2008).

\subsection{Subjects}

\subsubsection{Experiment 1}

12 right-handed subjects voluntarily participated in Experiment 1 . There were two females and 10 males, varying in age from 28 to 31 . Half of the subjects had previous experience with virtual environments.

\subsubsection{Experiment 2}

We invited four left-handed and eight right-handed subjects (no one was ambidextrous) in Experiment 2. 10 of them have worked with virtual environments and one was female. They had a mean age of 29.8 .

\subsection{Procedure}

\subsubsection{Experiment 1}

Experiment 1 was conducted to examine the effect of path length, width and curvature (three independent variables) on the movement time. We adopted a within-subject design, in which each independent variable was treated as a within-subject variable. For this, paths of varying length, width and curvature were used. Curvature is defined as $\rho=1 /$ radius, such that a path can be thought of as a segment on a circle of a given radius. The path was positioned in the $x y$-plane with the start of the path at the origin. The path lengths were chosen to be $0.24,0.30$ and $0.36 \mathrm{~m}$, so that participants needed to traverse a reasonable distance and were not required to move the body to accomplish

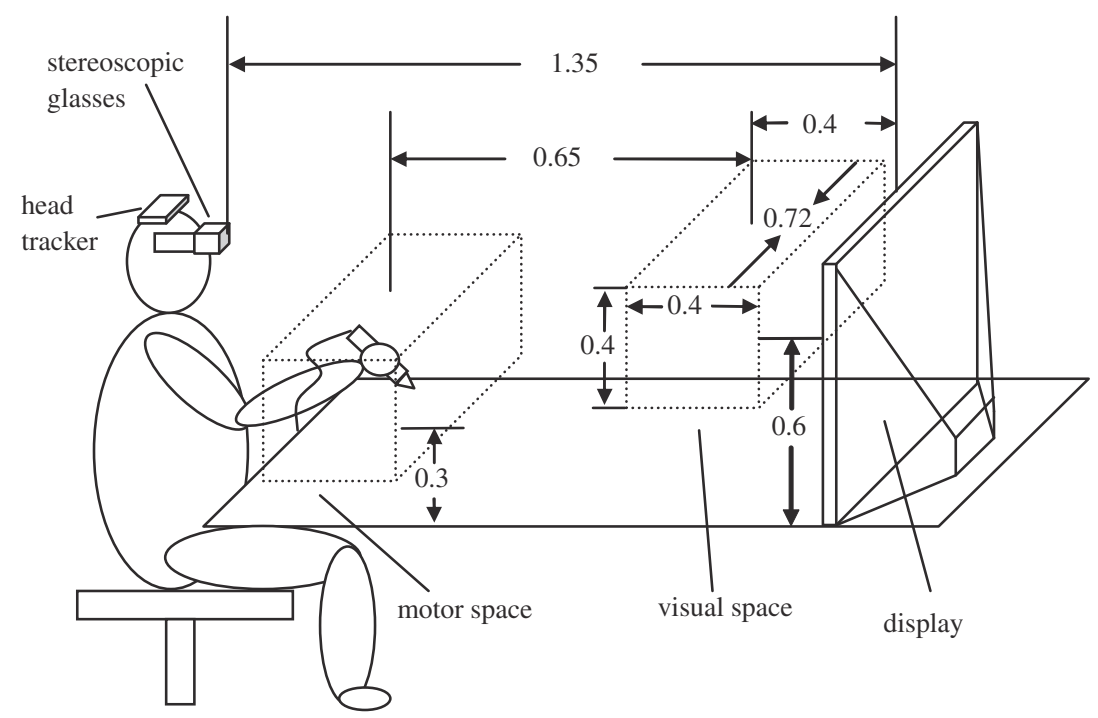

Fig. 4. The experimental setup (units: meter): Motor and visual space were not co-located, i.e. there is a horizontal offset of $0.65 \mathrm{~m}$ and a vertical offset of $0.3 \mathrm{~m}$ between the motor space and the visual space; Control-Display ratio was set to 1 . 
the mission, but the arm. The radius of the cursor ball was fixed to $0.005 \mathrm{~m}$, while the variable target ball had the radius of 0.010 and $0.015 \mathrm{~m}$, resulting in two path widths, 0.03 and $0.04 \mathrm{~m}$, respectively. Five typical curvature values $0,4,8,12,16 \mathrm{~m}^{-1}$ were well selected for the experiment (see Fig. 5), so that they were in arithmetic progression and corresponded to the circle of radius of inf (straight line), 0.2500, 0.1250, $0.0833,0.0625 \mathrm{~m}$. The maximum value was chosen in such a fashion that no circular path overlapped when different path lengths were designated. We also made sure that the difference between any two values was big enough to significantly affect the movement time. There were three repetitions per combination, resulting in $5 \times 3 \times 2 \times 3=90$ trials (curvatures $\times$ lengths $\times$ widths $\times$ repeats) per subject.

As defined by Accot and Zhai's model, if $C$ is a curved path and $W$ is a constant along the path, the index of difficulty for steering through this path is

$I D=\int_{C} \frac{d s}{W(s)}=\frac{L}{W}$

In Experiment 1, three path widths and two path lengths were used, resulting in six different $I D$ s.

\subsubsection{Experiment 2}

Experiment 2 was designed to examine the effect of path orientation relative to user's viewing direction on movement time. It was also a within-subject design, in which path orientation relative to the viewing direction was considered to be a within-subject variable. Other factors such as path length, width and curvature were fixed $(L=0.24 \mathrm{~m}, W=$ $0.04 \mathrm{~m}, \rho=8 \mathrm{~m}^{-1}$, see Fig. 5). We selected a representative path curvature from Experiment 1 as the one for Experiment 2 . The choice of the specific path length and width ensured that the path in Experiment 2 was easy to steer through, i.e. the path had the smallest $I D$ with the chosen $L$ and $W$ from Experiment 1, so that it kept the time of fulfilling the task to a minimum while focusing on the effect of observed variable (path orientation). The path was initially positioned in the $x y$-plane as shown in Fig. 6, with one end of the path placed at the origin, and then it was in turn rotated around the $y$-axis at an angle of $\beta$ degrees (see Fig. 7, left) and the $z$-axis of $\alpha$ degrees (see Fig. 7, right), respectively. In fact, the definition of angle $\alpha$ and $\beta$ is analogous to that of latitude and longitude on a globe. Depending on the selection of $\alpha$ and $\beta$, theoretically, we can position the other end of the path at any point on the globe, so that the path is placeable

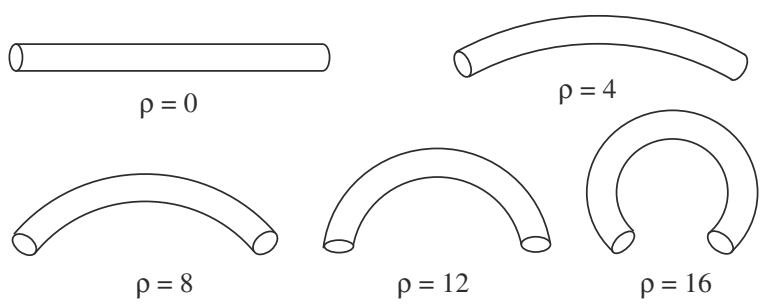

Fig. 5. Experiment 1: five paths of different constant curvatures.

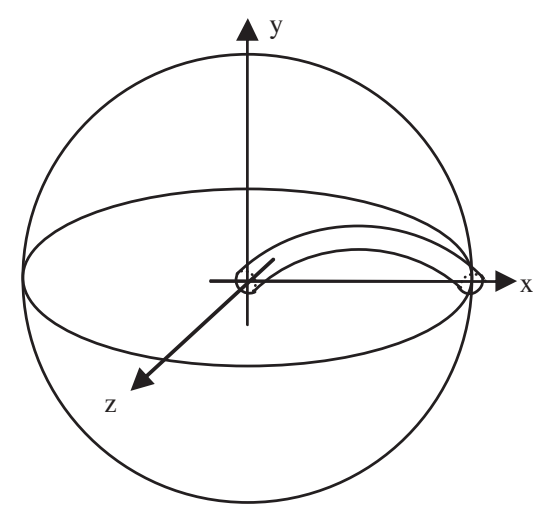

Fig. 6. Initial position of the path: The path was placed in $x y$-plane, with one end of the path at the origin and the other end at the point of intersection of equator and the $x$-axis.
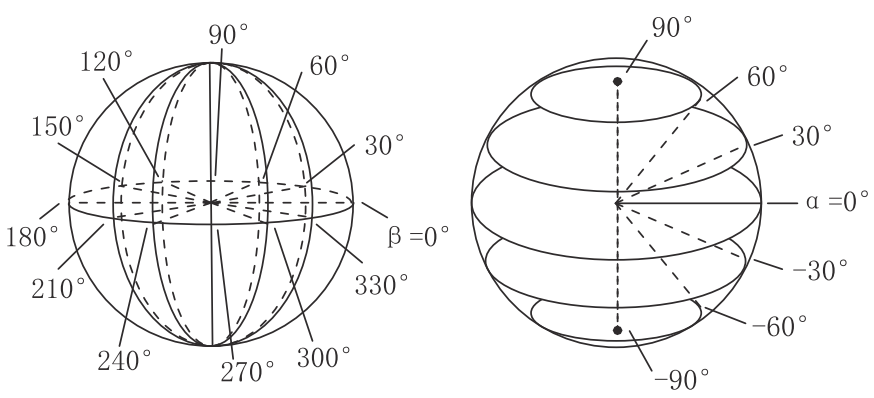

Fig. 7. Left: Rotating around the $y$-axis at an angle of $\beta$ degrees, which resembles the longitude on a globe. Right: Rotating around the $z$-axis at an angle of $\alpha$ degrees, which resembles the latitude on a globe.

Table 1

Specific rotating angles in latitude and longitude. A negative sign denotes a southern latitude. The south and north poles, i.e. $\alpha=-90^{\circ}$ and $90^{\circ}$, are just two points that cannot be further split into longitude.

\begin{tabular}{ll}
\hline Rotating orientation & Angles \\
\hline$\alpha$ (latitude) & $-90^{\circ},-60^{\circ},-30^{\circ}, 0^{\circ}, 30^{\circ}, 60^{\circ}, 90^{\circ}$ \\
$\beta$ (longitude) & $0^{\circ}, 30^{\circ}, 60^{\circ}, 90^{\circ}, 120^{\circ}, 150^{\circ}, 180^{\circ}, 210^{\circ}$, \\
& $240^{\circ}, 270^{\circ}, 300^{\circ}, 330^{\circ}$ \\
\hline
\end{tabular}

in any orientation in the $3 \mathrm{D}$ space. To obtain a representative end points sampling, the globe was divided into six in latitude with five planes (see Fig. 7, right) and further into 12 in longitude (see Fig. 7, left.) within each of the planes, except the two poles. The specific angles selected for latitude and longitude are enumerated in Table 1 . This resulted in $12 \times 5+2$ points on the globe, which consequently corresponded to 62 path orientations in 3D world. Each orientation was repeated three times, so that Experiment 2 required 186 trials per subject.

To counterbalance the interference and learning effect, in each experiment, the overall trials, including the repetitions, were given in a random order that was different between subjects. 


\section{Results}

The first observation from the data is that about $60-70 \%$ of the trials could not be completed by the subjects in a single steering operation. This means that the subjects often did not succeed in keeping their pen within the boundary of the tunnel, and that they needed to spend time to correct for this, and to bring the pen back within the boundary after wandering off too far. The instances where the pen entered and left the designated boundary were used to define subtrials, i.e. the time intervals during which actual steering was accomplished.

In Fig. 8, we have plotted the cumulative histogram of the subtrial path lengths. The fact that path lengths other than the ones specified in the experimental setup occur is evidence for our above claim. For this, we have partitioned and analyzed the data such that the total time of a trial is equal to the steering time of the subtrials plus the correction time:

$T_{\text {total }}=T_{\text {steering }}+T_{\text {correction }}$

where $T_{\text {steering }}=\sum T_{\text {subtrials }}$. Strictly speaking, the steering law as specified by Accot and Zhai should hold for subtrials.

Instead of looking for a linear relationship between the time $T$ taken for a subtrial and the index of difficulty $I D=$ $L / W$, where $L$ is the path length crossed during the subtrial, we propose an alternative analysis where we look for a linear relationship between $\log (T)$ and $\log (I D)$. In Appendix (a), we provide the reasons for this choice. Unless explicitly stated otherwise, the base of the logarithm used in this paper is 10 . We consider equation of the form

$\log T=a+b \log \frac{L}{W}=a+b \log I D$

to statistically test the steering law. This equation corresponds to a power-law relationship

$T=a^{\prime} I D^{b}$

between time $T$ and index of difficulty $I D$ (see Appendix (b) for reasoning). If the exponent $b$ does not differ significantly from one, then this can be considered as evidence that the steering law is a statistically valid description of the data. If the exponent $b$ differs significantly from one, then this can be considered as evidence that the steering law is not the best possible description of the data.

The effect of curvature can be included by extending the model to

$\log T=a+b \log \frac{L}{W}+c \rho+d \rho \log \frac{L}{W}$

in the case where we want to include interaction between curvature $\rho$ and $I D$, or

$\log T=a+b \log \frac{L}{W}+c \rho$

if we can assume that such interaction term is negligible.

Before performing the ANOVAs, the repetitions of the trials under the same condition were averaged within each subject, which were further averaged out between subjects for the regression analysis below.

\subsection{Experiment 1: the effect of path length, width and curvature}

The regression parameter estimates using the model defined by Eq. (9) to fit the subtrial data are given in Table 2, in which the estimated power $b=0.59$ is substantially different from one. The fact that the $95 \%$ confidence interval of $d$ in Eq. (8) includes zero ([-0.013,0.015]) indicates that no statistical evidence is found for the interaction term. Note in Table 2 that there is indeed a small, but statistically significant effect of the curvature (the

Table 2

Regression parameter estimates on subtrial time (fitting onto Eq. (9)).

\begin{tabular}{lrl}
\hline Coef. & \multicolumn{1}{l}{ Value } & {$[95 \%$ conf. interval $]$} \\
\hline$a$ & -0.318 & {$[-0.337,-0.299]$} \\
$b$ & 0.592 & {$[0.573,0.612]$} \\
$c$ & 0.005 & {$[0.004,0.006]$} \\
\hline
\end{tabular}

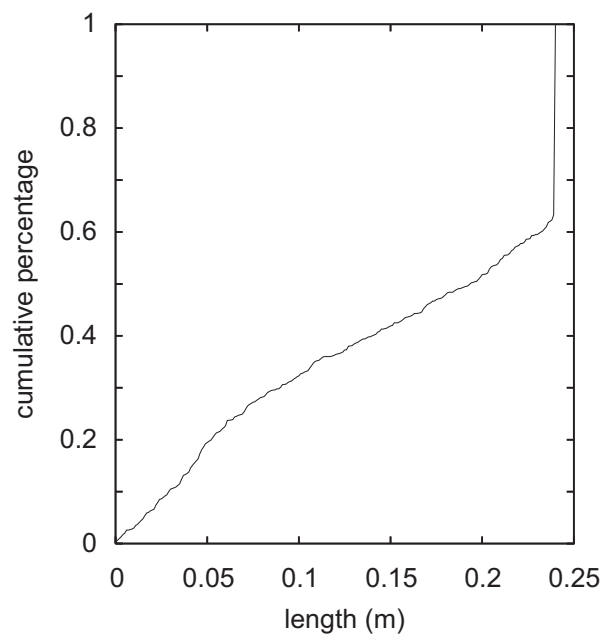

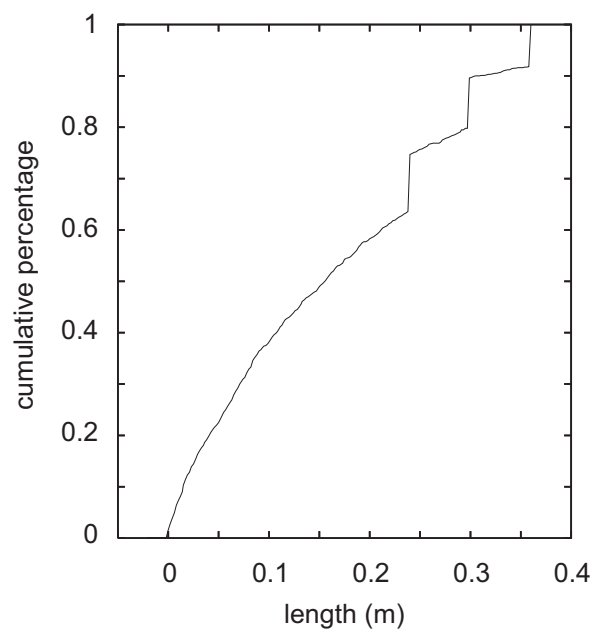

Fig. 8. Cumulative histograms of path lengths for subtrials: Experiment 1 (left), Experiment 2 (right). 
95\% confidence interval does not include zero). The interpretation of Eq. (9) in terms of the above-specified power-law (Eq. (7)) is that the power $b$ is independent of curvature, and only the factor $a^{\prime}$ is curvature-dependent.

The correspondence between the raw data and the model can be assessed on the left plot of Fig. 9, where only the effect of $I D$ is illustrated. The right plot illustrates the effects of both $I D$ and $\rho$ on the steering time. Raw data are plotted as asterisks with error bars, representing the corresponding $95 \%$ confidence intervals, while the dotted diagonal lines are the related steering models. The influence of curvature is evident as a shift in the curves (the slope is the same), or equivalently, a percentage change on the time required that is independent of $I D$.

The fact that the steering law is not able to closely approximate the actual steering taking place during subtrials does not exclude the possibility that the steering law might be an appropriate description for the total performance time. Indeed, the regression parameter estimates in Table 3, and the data shown on the left of Fig. 10 indicate that a powerlaw (Eq. (7)) with an exponent $b$ close to one, and a factor $a^{\prime}$ that increases with curvature, provide a good description for the complete trial steering time.

What can be concluded from the data on the right plot of Fig. 10 is that the correction time required to successfully complete the task increases rapidly with increasing $I D$ and $\rho$. More precisely, a power law (Eq. (7)) with an exponent in the range 2.5-3.0 (i.e. much steeper than a linear increase with $I D)$ is needed to describe this relationship.

\subsection{Experiment 2: the effect of path orientation relative to the viewing direction}

In Experiment 2, we have positioned the path of fixed path length, width and curvature in such a way that it was first rotated around the $y$-axis at an angle of $\beta$ degrees, and then around the $z$-axis of $\alpha$ degrees. Analogous to the analysis in the previous section, data have been transformed logarithmically to observe an approximate Gaussian distribution.

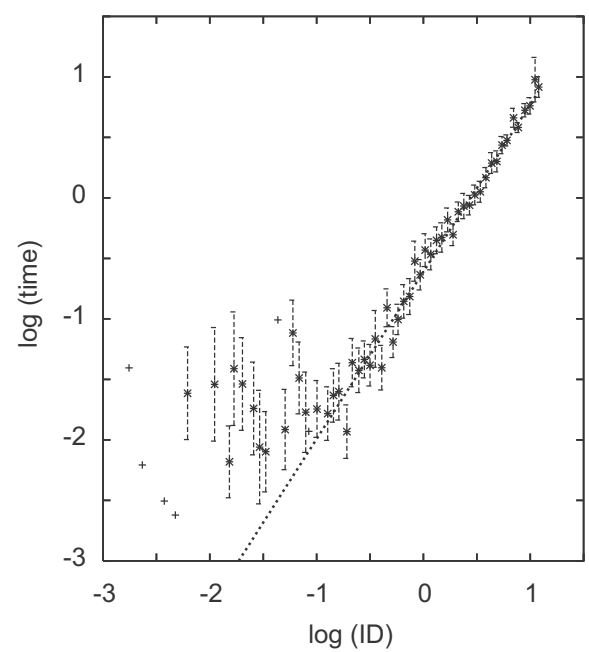

Fitting the subtrial time of Experiment 2 onto the model described in Eq. (6), we find a power law (Eq. (7)) with an exponent $b=0.82$. The corresponding data are illustrated in Fig. 11.

We have performed a repeated measures ANOVA to examine the effect of $\alpha$ on the steering time. The corresponding result is shown in Fig. 12. An evidence of significant difference $(F(4,44)=8.345, p<0.0001)$ has been found between the average steering time of various $\alpha$ values. Generally speaking, there is a U-shaped influence where paths of rotating around the $z$-axis $0^{\circ}$ (the paths whose end positions fall on the equator, see Fig. 7 on the right) always result in a valley in the steering time. The steering time increases as the end positions of the paths deviate from the equator and the further the longer. There also tends to be a symmetric effect on the steering to the south and the north (negative and positive $\alpha$ values), indicating that function $\cos (\alpha)$ may play an important role in modeling the effect of $\alpha$ on the steering time.

A similar analysis has been done for the influence of $\beta$ on the steering time. Fig. 13 illustrates the repeated measures ANOVA result. There is also a significant difference $(F(11,121)=19.498, p<0.0001)$ between the average steering time for varying $\beta$ values. As depicted, the $\mathrm{W}$-like curve derived from connecting all the asterisks tends to periodically oscillate up and down as $\beta$ grows. In general, it has two significantly different local minima at paths of rotating around the $y$-axis $90^{\circ}$ and $270^{\circ}$, i.e. the paths parallel to the viewing direction (see Fig. 7 on the left), and two local

Table 3

Regression parameter estimates on complete trial steering time (fitting onto Eq. (9)).

\begin{tabular}{lrl}
\hline Coef. & Value & {$[95 \%$ conf. interval $]$} \\
\hline$a$ & -0.634 & {$[-0.687,-0.580]$} \\
$b$ & 0.969 & {$[0.913,1.026]$} \\
$c$ & 0.008 & {$[0.007,0.009]$} \\
\hline
\end{tabular}

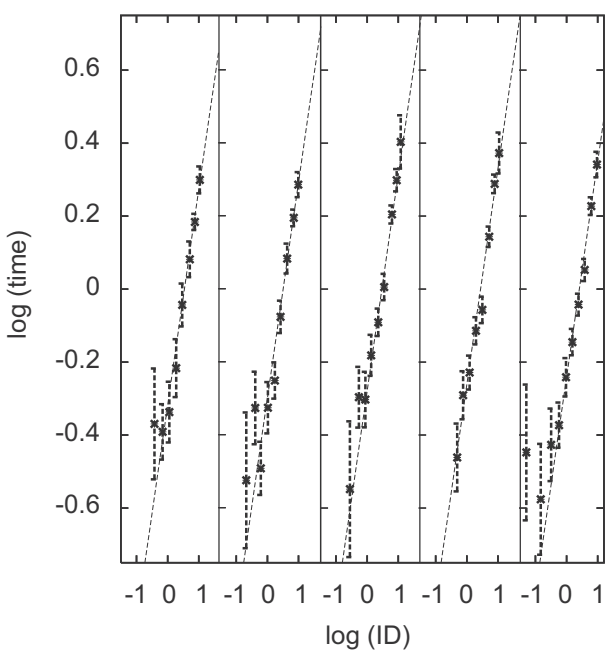

Fig. 9. Subtrial time from Experiment 1 fits onto different steering models. Left: Logarithmic subtrial time as a function of log(ID) (Eq. (6)). Right: Logarithmic subtrial time as a function of $\log (I D)$ for the five different values of curvature $\rho$ (Eq. (9), see Table 2 for regression results.). 

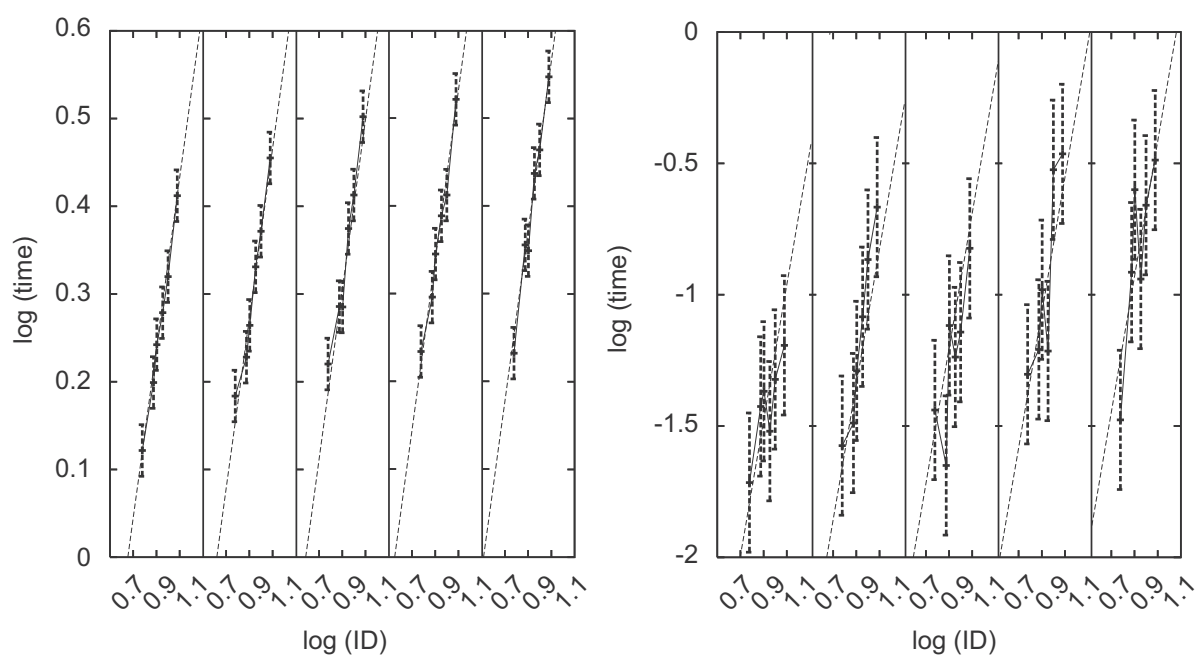

Fig. 10. Left: Logarithmic complete trial steering time as a function of $\log (I D)$ fits onto Eq. (9), see Table 3 for regression results. Right: Logarithmic complete trial correction time as a functions of $\log (I D)$ fits onto Eq. (9).

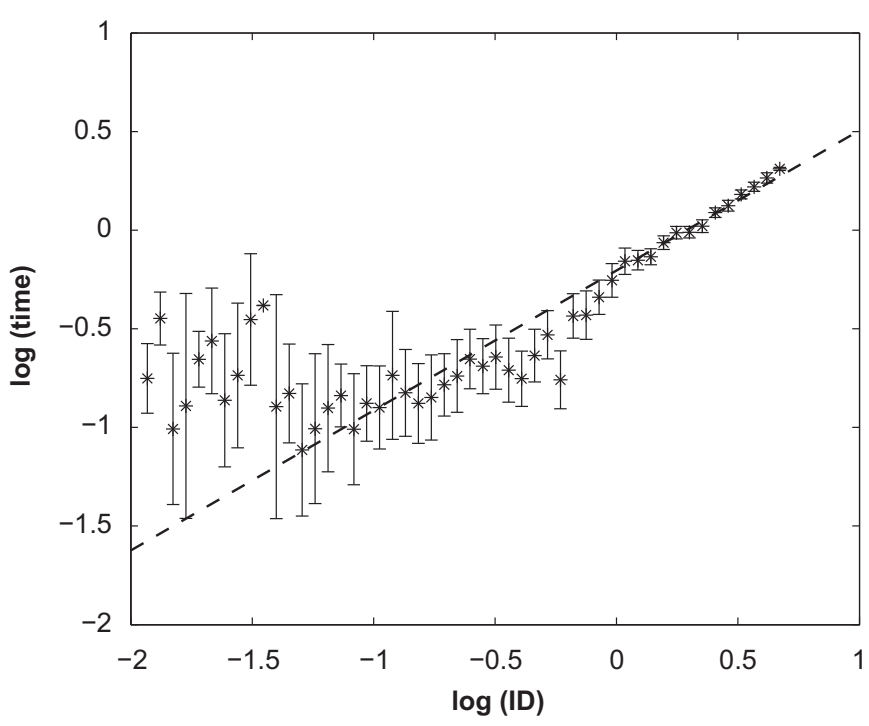

Fig. 11. Experiment 2: Logarithmic subtrial time as a function of $\log (I D)$ fits onto Eq. (6).

maxima in the vicinity of $0^{\circ}$ (or $330^{\circ}$ ) and $180^{\circ}$, i.e. the paths perpendicular to the viewing direction. This seems to resemble the characteristics of the function of $\sin (\beta)$ or $\cos (\beta)$ except with varying peaks and valleys, which might be described optimally using a Fourier series expansion.

Given the components that the model may comprise with the effect of $\alpha$ and $\beta$ separately, we propose a model to combine the effect of $\alpha$ and $\beta$ in Eq. (10):

$\log T=a+b \cos (\alpha)+c \sin (\alpha)+d \cos (\beta)+e \sin (\beta)+f \cos (2 \beta)+g \sin (2 \beta)$

where all the potential terms are added to construct the model, including a one-level Fourier series expansion on $\alpha$ and a two-level Fourier series expansion on $\beta$ (see discussion for the reasons).

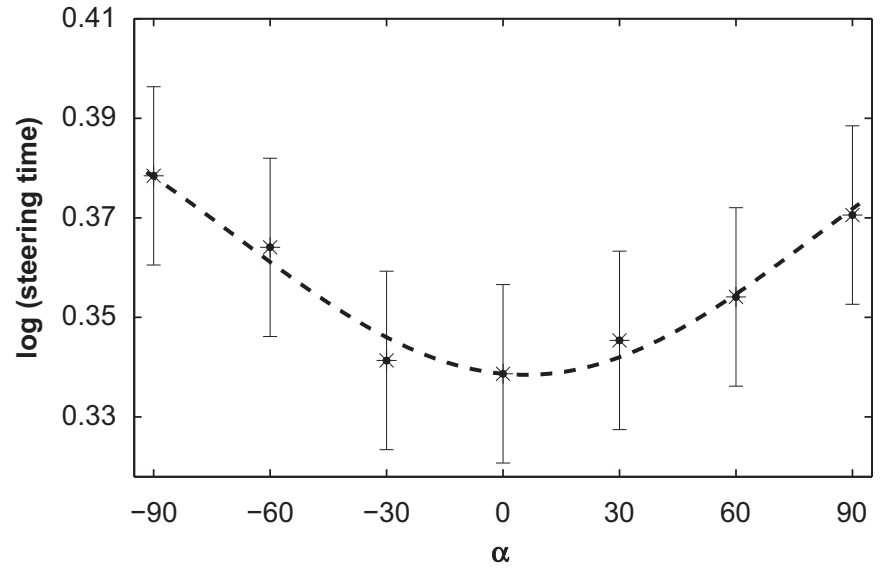

Fig. 12. The repeated measures ANOVA result on the steering time of various $\alpha$ values. The asterisks represent the average of logarithmic steering time of the related $\alpha$ values and the error bars represent the $95 \%$ confidence intervals, calculated using the method in Masson and Loftus (2003). The dotted curve is the model $\log (T)=a+b \cos (\alpha)+c \sin (\alpha)$.

The regression parameter estimates using the model of Eq. (10) have been specified in Table 4. There is conclusive evidence for the existence of the term $\cos (\alpha), \cos (\beta)$, $\sin (\beta), \cos (2 \beta)$ and $\sin (2 \beta)$. It has been verified that regressions with one of these terms dropped led to significantly worse results. The fact that the absolute values of the coefficients $b$ to $g$ in Table 4 are quite small indicates that path orientation may slightly influence the steering time, though the effect is statistically evident. No evidence, however, could be found for the term $\sin (\alpha)$ due to the inclusion of 0 in the $95 \%$ confidence interval of coefficient $c$. This is predictable from the results demonstrated in Fig. 12. Therefore, Eq. (10) could be further simplified as

$\log T=a+b \cos (\alpha)+c \cos (\beta)+d \sin (\beta)+e \cos (2 \beta)+f \sin (2 \beta)$ 


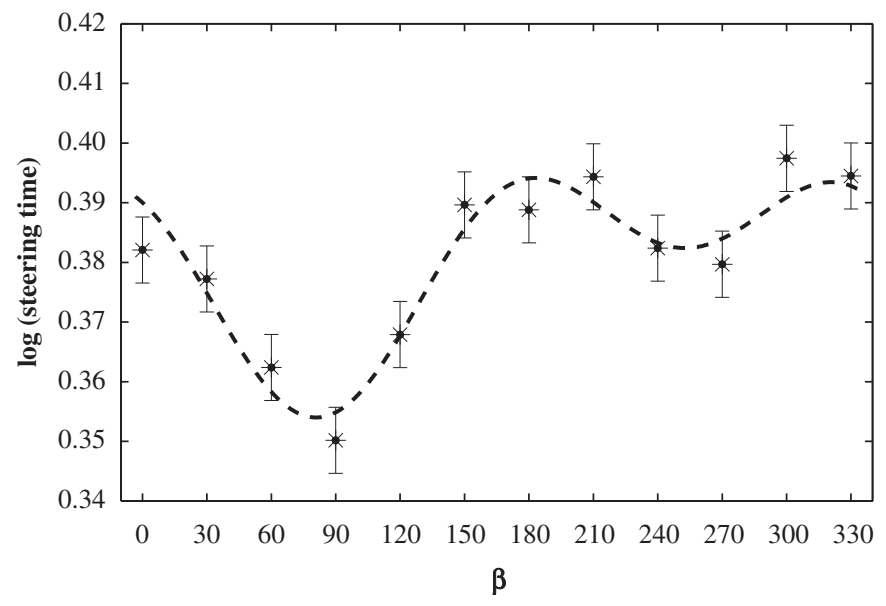

Fig. 13. The repeated measures ANOVA result on the steering time of various $\beta$ values. The asterisks are the raw data processed logarithmically, while the dotted curve is the model $\log (T)=a+b \cos (\beta)+c \sin (\beta)+$ $d \cos (2 \beta)+e \sin (2 \beta)$.

Table 4

Regression parameter estimates on steering time (fitting onto Eq. (10)).

\begin{tabular}{lrl}
\hline Coef. & \multicolumn{1}{l}{ Value } & {$[95 \%$ conf. interval $]$} \\
\hline$a$ & 0.3757 & {$[0.3668,0.3845]$} \\
$b$ & -0.0486 & {$[-0.0602,-0.0370]$} \\
$c$ & -0.0042 & {$[-0.0085,0.0001]$} \\
$d$ & 0.0297 & {$[0.0258,0.0336]$} \\
$e$ & -0.0044 & {$[-0.0084,-0.0004]$} \\
$f$ & -0.0235 & {$[-0.0274,-0.0196]$} \\
$g$ & 0.0105 & {$[0.0065,0.0145]$} \\
\hline
\end{tabular}

\section{Discussion}

There are a number of issues that are raised by the experiments.

The first issue is how we better model the path steering with the known influential parameters, i.e. path length, width, curvature, orientation, etc. The index of difficulty of a steering task, according to Accot and Zhai's model, is only governed by the length and the width of the steering path. Consequently, paths 1 and 2 shown at the top of Fig. 1, of the same path width and length, were considered to have the same $I D$, although this is somewhat counterintuitive. The failure of taking the path curvature and the orientation into account significantly reduces its capacity when applied to different steering tasks. By including the effect of curvature as in Eq. (9) and the effect of orientation in Eq. (11), we provide the possibility to compare the steering efficiency on the paths of different shapes and placed in various orientations. It is beyond the scope of Accot and Zhai's steering law in the sense that the steering on straight and circular paths can be dealt with together and particularly, 3D manipulation steering tasks where path orientation plays a role have been addressed. We propose to consider a combination of
Eqs. (9) and (11):

$$
\begin{aligned}
\log T= & a+b\left(\log \frac{L}{W}+c \rho+d \cos (\alpha)+e \cos (\beta)+f \sin (\beta)\right. \\
& +g \cos (2 \beta)+h \sin (2 \beta))
\end{aligned}
$$

where $I D$ is redefined as $\log L / W+c \rho+d \cos (\alpha)+e \cos (\beta)+$ $f \sin (\beta)+g \cos (2 \beta)+h \sin (2 \beta)$, introducing the influence of curvature $(\rho)$ and orientation ( $\alpha$ and $\beta$ ) of the steering path, together with length $(L)$ and width $(W)$. It is not difficult to discover to what extend the seven terms play the roles in affecting the steering time by examining the related coefficients before the terms in the new ID. However, since Eq. (12) is derived from two independent experiments, the way that the effect of path curvature and orientation combines needs to be verified in the following work. In addition, the model was developed particularly for the path of fixed curvature and width, e.g. equal width linear $(\rho=0)$ or circular $(\rho=1 / R)$ paths. The message conveyed from the model is that there is a conclusive effect of path curvature and orientation on path steering tasks. A more powerful model can be developed for a general path whose curvature and width vary along the path in the future.

The second issue has to do with how subjects actually decompose a steering movement into sub-movements, i.e. to get more insight into what the elementary units are in the overall movement. The subtrials that we have used in this paper are obviously not the optimal way to segment the overall trial, as a subtrial can potentially consists of several submovements, in agreement with Accot and Zhai's assumption. The only way to actually resolve this is to adopt a more objective way of subdividing the (sub)trials, for instance using a similar parsing method as was adopted for analyzing directed movements (Nieuwenhuizen et al., 2009). Since the movements of the pen and the cursor ball were logged during the experiment, such analysis is feasible and planned in the near future. For now, we could hypothesize, also based on the experimentally observed value for the power function, that we expect more analogy with goal directed movements than is implied by the steering law and its theoretical derivation.

The third issue is that we have to balance a trade-off between the complexity of the function used to model the effect of path orientation on the steering time and the fitting accuracy. With a two-level Fourier series expansion, we could use a minimum number of cosine and sine functions to statistically describe the intrinsic characteristics of the effect of varying path orientation in $\beta$ on the steering time while keeping a relatively high fitting accuracy. A one-level Fourier series expansion is sufficient to depict the effect of varying $\alpha$ on the steering time as shown in Fig. 12. The fact, however, is that there are possibilities that the effect of orientation could be more precisely modeled with multiple-level (more than two) Fourier series expansions, but with a much more complicated form.

The last issue is how the handedness of the subjects affects the steering time on paths of different orientations. In a previous paper (Liu et al., 2010), we have observed an asymmetric effect, 


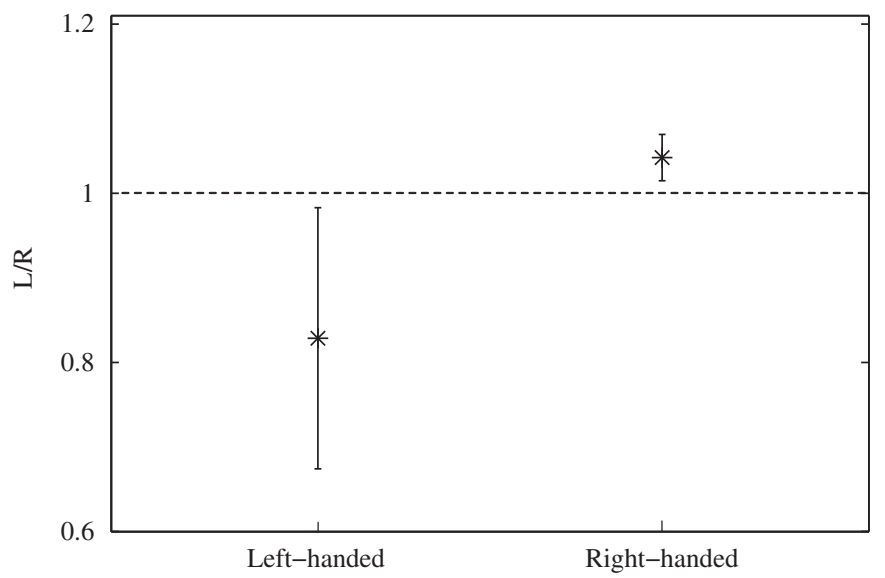

Fig. 14. The between-subjects ANOVA result between left-handed and right-handed subjects on the ratio of left district steering time and right district steering time. The left district includes the paths of rotating around $y$-axis $0^{\circ}, 30^{\circ}, 60^{\circ}, 300^{\circ}, 330^{\circ}$, while the right district is composed of paths of $120^{\circ}, 150^{\circ}, 180^{\circ}, 210^{\circ}, 240^{\circ}$. See Fig. 7 on the left.

i.e. moving to the left requires more time than moving to the right. This might be attributed to the handedness, since all subjects reported the right hands as the dominant hands in that study and moving to the right seems to be easier than to the left for right-handed subjects. In this work, we invited four lefthanded subjects besides eight right-handed subjects and none of them was ambidextrous. Instead of comparing the absolute steering time of moving to the left and right between the lefthanded and right-handed groups, we have performed a between-subjects ANOVA for the ratio of steering time of moving to the left (the average steering time to navigate through paths of $\beta=0^{\circ}, 30^{\circ}, 60^{\circ}, 300^{\circ}, 330^{\circ}$ ) divided by that to the right (the average steering time to navigate through paths of $\beta=120^{\circ}$, $\left.150^{\circ}, 180^{\circ}, 210^{\circ}, 240^{\circ}\right)$ to minimize the difference between individuals. Fig. 14 illustrates the result between left-handed and right-handed groups. It can be seen that the mean ratio of lefthanded group is significantly smaller than that of right-handed group. In addition, the mean ratio of left-handed group is smaller than 1, indicating that for left-handed subjects steering to the left requires less time than steering to the right. On the contrary, the fact that the ratio is greater than 1 in right-handed group specifies that it is faster to steering through paths diverting to the right than to the left. This is the conclusive evidence showing that subjects' handedness plays a part in the steering of paths with varying orientations. Therefore, we suggest that handedness should be seriously considered as a between-subject factor when designing experiments of path steering tasks.

\section{Conclusion}

We have addressed Accot and Zhai's question if the steering law could also be used to model the performance of 3D manipulations tasks. The ball and tunnel task is somewhat simpler than the ring and wire task proposed by Accot and Zhai, since the cursor ball is orientation-independent, whereas the ring must be oriented in such a way that it does not intersect the wire.
We have experimentally demonstrated that the steering law is able to predict the overall performance of the ball and tunnel task. The data analysis confirms the steering law (time proportional to $L / W$ ) for complete trial steering time, but not for subtrial time. Furthermore, we have also shown that other factors are significant in affecting the steering time, besides path length and width. There is a systematic effect of the curvature on both the total and steering time. This effect can accurately be modeled as a percentage increase in time for increasing curvatures. The effect of varying path orientation with respect to the viewing direction periodically influences the path steering time in such a way that it requires less time when subjects steer parallel to the viewing direction than perpendicular. Additionally, the effect of handedness plays a part in influencing the steering time in 3D manipulation tasks as well. Steering to the left is more efficient than to the right for left-handed subjects, which is exactly opposite for the right-handed subjects.

\section{Appendix A}

\section{A.1. Appendix (a)}

Since the data analysis that we performed in this paper is somewhat unusual, it is useful to summarize the reasons why we think that an analysis based on $\log ($ time $)$ is superior over an analysis based on time.

1. Methodology: a regression analysis assumes Gaussian noise with (approximate) constant variance; this is clearly not the case of time where the observed variance often increases with time (and moreover, time cannot be Gaussian distributed as it is restricted to being positive). Looking at the cumulative histograms of the subtrial times in Fig. 15, we find that the observed times have an asymmetrical distribution, which clearly deviates from the Gaussian distribution assumption made in most regression (and ANOVA) analysis methods. A one-sample Kolmogorov-Smirnov test has been performed to compare the empirical data to a normal distribution fitting onto the data. The null hypothesis, i.e. the empirical data have a normal distribution, was rejected $(h=1)$ at the $5 \%$ significance level in each experiment. Taking the logarithm of time usually helps to bring the observed time distributions closer to such a Gaussian distribution, as is evidenced by the distributions in Fig. 16, where the Kolmogorov-Smirnov test indicates that the transformed data come from a normal distribution $(h=0)$ in each experiment.

2. Modeling: linear regression on $\log ($ time $)$ corresponds to a different class of functions (power functions that pass through the origin) than linear regression on time; as the regression lines on time in our data were observed to pass (approximately) through the origin, the power functions comprise a more general class of models.

3. Interpretation: in order to test the steering law, a test to determine whether or not time varies linearly with $L / W$ is needed; by considering a larger class of models (power functions), we can translate this in a statistical test on the 

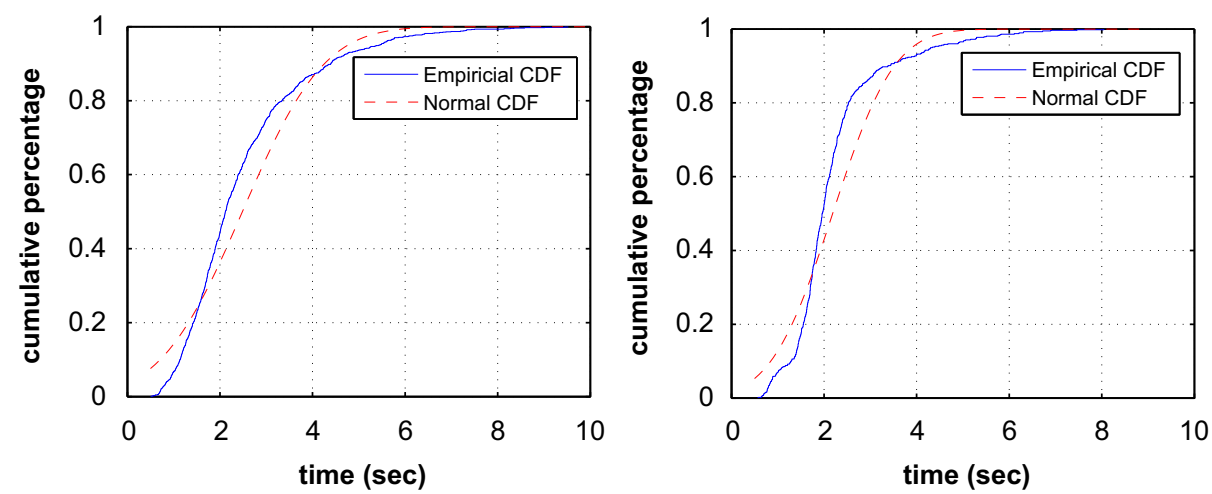

Fig. 15. Cumulative histograms of subtrial time: Experiment 1 (left), Experiment 2 (right). Both histograms (empirical CDF) show strong deviation from the Gaussian distribution fitting onto the data (normal CDF: the same mean and standard deviation to the empirical data) in both experiments.
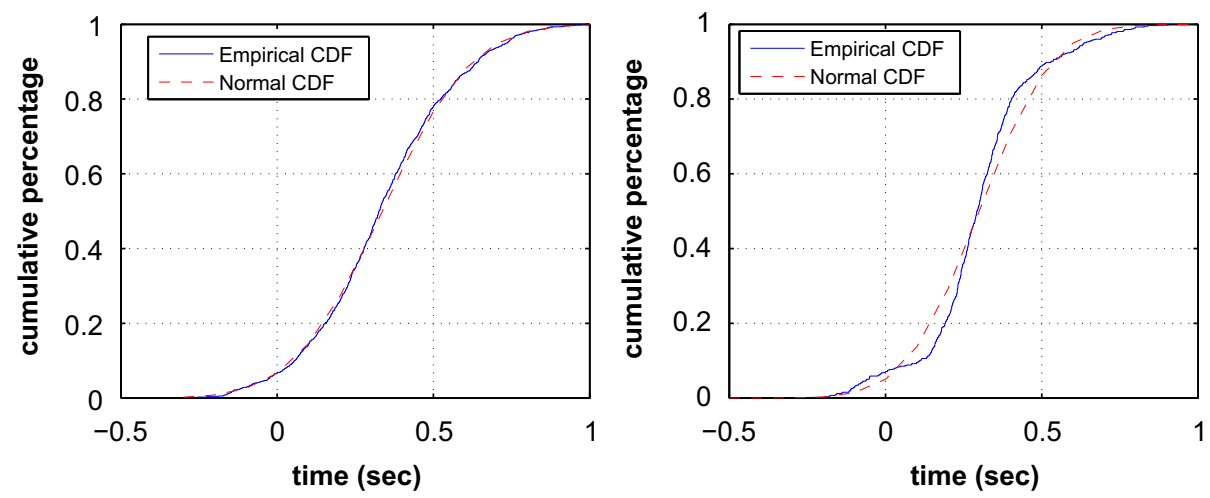

Fig. 16. Cumulative histograms of subtrial logarithm time: Experiment 1 (left), Experiment 2 (right). After transformed logarithmically, subtrial time (empirical CDF) approximately follows the Gaussian distribution fitting onto the data (normal CDF: the same mean and standard deviation to the empirical data) in both experiments.

power (testing whether or not it is significantly different from 1).

4. Communication: the different slopes that are found in linear regression lines on time can be translated into a shift for the regression lines on $\log ($ time) (a percentage change, see Fig. 9 on the right); this is easier to communicate and moreover, also shows that the effect of curvature is constant (in percentage) across $I D \mathrm{~s}$.

\section{A.2. Appendix (b)}

$$
\begin{aligned}
& \log T=a+b \log I D=a+\log I D^{b} \\
& \Downarrow \\
& 10^{\log T}=10^{a+\log I D^{b}} \\
& \Downarrow \\
& T=10^{a} 10^{\log I D^{b}} \\
& \Downarrow \\
& T=a^{\prime} I D^{b} \quad\left(a^{\prime}=10^{a}\right)
\end{aligned}
$$

\section{References}

Accot, J., Zhai, S., 1997. Beyond Fitts' law: models for trajectory-based HCI tasks. In: Proceedings of the SIGCHI Conference on Human Factors in Computing Systems, pp. 295-302.
Accot, J., Zhai, S., 1999. Performance evaluation of input devices in trajectorybased tasks: an application of the steering law. In: Proceedings of the SIGCHI Conference on Human Factors in Computing Systems, pp. $466-472$.

Accot, J., Zhai, S., 2001. Scale effects in steering law tasks. In: Proceedings of the SIGCHI Conference on Human Factors in Computing Systems, pp. 1-8.

Accot, J., Zhai, S., 2003. Refining Fitts' law models for bivariate pointing. In: Proceedings of the SIGCHI Conference on Human Factors in Computing Systems, pp. 193-200.

Blanch, R., Guiard, Y., Beaudouin-Lafon, M., 2004. Semantic pointing: improving target acquisition with control-display ratio adaptation. In: Proceedings of the SIGCHI Conference on Human Factors in Computing Systems, pp. 519-526.

Boritz, J., Booth, K.S., 1997. A study of interactive 3D point location in a computer simulated virtual environment. In: Proceeding of the ACM Symposium on Virtual Reality Software and Technology, pp. 181-187.

Elmqvist, N., Fekete, J.-D., 2008. Semantic pointing for object picking in complex 3D environments. In: Proceedings of Graphics Interface, pp. 243-250.

Fitts, P.M., 1954. The information capacity of the human motor system in controlling the amplitude of movement. Journal of Experimental Psychology 47 (6), 381-391.

Grossman, T., Balakrishnan, R., 2004. Pointing at trivariate targets in 3D environments. In: Proceedings of the SIGCHI Conference on Human Factors in Computing Systems, pp. 447-454.

Grossman, T., Hinckley, K., Baudisch, P., Agrawala, M., Balakrishnan, R., 2006. Hover widgets: using the tracking state to extend the capabilities of pen-operated devices. In: Proceedings of the SIGCHI Conference on Human Factors in Computing Systems, pp. 861-870. 
Kattinakere, R.S., Grossman, T., Subramanian, S., 2007. Modeling steering within above-the-surface interaction layers. In: Proceedings of the SIGCHI Conference on Human Factors in Computing Systems, pp. 317-326.

Kopper, R., Bowman, D.A., Silva, M.G., McMahan, R.P., 2010. A human motor behavior model for distal pointing tasks. International Journal of Human-Computer Studies 68 (10), 603-615.

Liu, L., Martens, J.-B., van Liere, R., 2010. Revisiting path steering for 3D manipulation tasks. In: Proceedings of the IEEE Symposium on 3D User Interfaces, pp. 39-46.

Liu, L., van Liere, R., 2009. Designing 3D selection techniques using ballistic and corrective movements. In: Proceedings of Eurographics Symposium on Virtual Environments, pp. 1-9.

Liu, L., van Liere, R., Nieuwenhuizen, C., Martens, J.-B., 2009. Comparing aimed movements in the real world and in virtual reality. In: Proceeding of the IEEE Virtual Reality, pp. 219-222.

MacKenzie, I.S., 1992. Fitts' law as a research and design tool in humancomputer interaction. Human-Computer Interaction 7, 91-139.

MacKenzie, I.S., Buxton, W.A.S., 1992. Extending Fitts' law to twodimensional tasks. In: Proceedings of the SIGCHI Conference on Human Factors in Computing Systems, pp. 219-226.

MacKenzie, I.S., Sellen, A., Buxton, W.A.S., 1991. A comparison of input devices in element pointing and dragging tasks. In: Proceedings of the SIGCHI Conference on Human Factors in Computing Systems 1991: Reaching Through Technology, pp. 161-166.

Masson, M.E., Loftus, G.R., 2003. Using confidence intervals for graphically based data interpretation. Canadian Journal of Experimental Psychology 57, 203-220.
Meyer, D.E., Abrams, R.A., Kornblum, S., Wright, C.E., Smith, J.E.K., 1988. Optimality in human motor performance: ideal control of rapid aimed movements. Psychological Review 95, 340-370.

Murata, A., Iwase, H., 2001. Extending Fitts' law to a three-dimensional pointing task. Human Movement Science 20, 791-805.

Naito, S., Kitamura, Y., Kishino, F., 2004. Steering law in an environment of spatially coupled style with matters of pointer size and trajectory width. In: Computer Human Interaction Sixth Asia Pacific Conference-APCHI 2004, vol. 3101, pp. 305-316.

Nieuwenhuizen, C., Liu, L., van Liere, R., Martens, J.-B., 2009. Insight into 3D goal-directed movements through a division into meaningful movement phases. IEEE Computer Graphics and Applications 29 (6).

Pastel, R., 2006. Measuring the difficulty of steering through corners. In: Proceedings of the SIGCHI Conference on Human Factors in Computing Systems, pp. 1087-1096.

Steed, A., 2008. A simple method for estimating the latency of interactive, real-time graphics simulations. In: Proceedings of the 2008 ACM Symposium on Virtual Reality Software and Technology, pp. 123-129.

Worden, A., Walker, N., Bharat, K., Hudson, S., 1997. Making computers easier for older adults to use: area cursors and sticky icons. In: Proceedings of the SIGCHI Conference on Human Factors in Computing Systems, pp. 266-271.

Zhai, S., Accot, J., Woltjer, R., 2004. Human action laws in electronic virtual worlds: an empirical study of path steering performance in VR. Presence: Teleoperators and Virtual Environments 13 (2), 113-127. 